\title{
INTERNET REACTOR LABORATORY: A NUCLEAR REACTOR PHYSICS AND ENGINEERING EDUCATION MODALITY FOR THE $21^{\text {st }}$ CENTURY
}

\author{
A. I. Hawari, S. A. Lassell, G. I. Gibson \\ Nuclear Reactor Program, Department of Nuclear Engineering \\ North Carolina State University, Raleigh, NC 27695, USA \\ ayman.hawari@ncsu.edu
}

\begin{abstract}
The "second generation" Internet Reactor Laboratory (IRL) has been installed and is currently functional at the PULSTAR reactor of the Nuclear Reactor Program (NRP) at North Carolina State University (NCSU). The NRP has offered IRL based reactor physics experiments and training to external academic institutions and organizations since 2004. In 2016, the United States and the Republic of Vietnam entered into an Administrative Arrangement under Section 123 of the Atomic Energy Act, with a goal of enhancing nuclear training and education for Vietnamese engineering students. Funding was provided by the DOE to upgrade the PULSTAR IRL infrastructure to state-of-the-art remote data acquisition and video teleconferencing capabilities supporting content sharing across multiple remote user platforms. Subsequently, a Cisco Telepresence and LabView reactor data acquisition system were installed and commissioned at the PULSTAR facility. Using this "second generation" system, and during IRL sessions, up to 100 remote students may interact with the control room staff via the Telepresence system using their mobile device or PC, and view 130 channels of live reactor telemetry via the remote client LabView application while recording the data for subsequent review and analysis. LabView graphical user interfaces (GUI) are provided for each IRL module, and incorporate embedded supporting documents such as schematics and diagrams that aid in illustrating associated technical details and concepts. Consequently, the educational experience offered to remote students and trainees is enhanced and assured to be of the same quality as offered to onsite students/trainees at NCSU.
\end{abstract}

\section{KEYWORDS: NRP, PULSTAR, Internet Reactor Laboratory}

\section{INTRODUCTION}

The 1 MW PULSTAR Reactor is the fourth nuclear research reactor built on the campus at N.C. State University. Over the past sixty-six years, the Nuclear Reactor Program has overseen the design and construction of several reactors at N.C. State and has developed an associated community of multidisciplinary users to make full use of their educational and research capabilities. Today the PULSTAR Reactor carries on this mission, providing a suite of educational, research, and service capabilities that serve the local, national and international academic, government and industrial communities. Over the past 18 years, a focus on the development and enhancement of state-of-the-art beamport irradiation facilities, inpool irradiation facilities, and educational and operational infrastructure have resulted in a quadrupling of facility utilization across all user groups [1]. Beamport facilities developed include an Intense Positron Beam with two Positron Annihilation Lifetime Spectrometers (PALS) and with associated Bulk ${ }^{22} \mathrm{Na}$ source-based PALS, a Neutron Powder Diffraction Facility, a Neutron Imaging Facility, a Fission Gas Release and Monitoring Loop [2], and an Ultracold Neutron Source (see Fig. 1). Operational infrastructure has also been upgraded to support an increase in licensed power to $2 \mathrm{MW}$ (currently pending NRC approval) and improve the reliability, safety and security of plant systems [3]. 


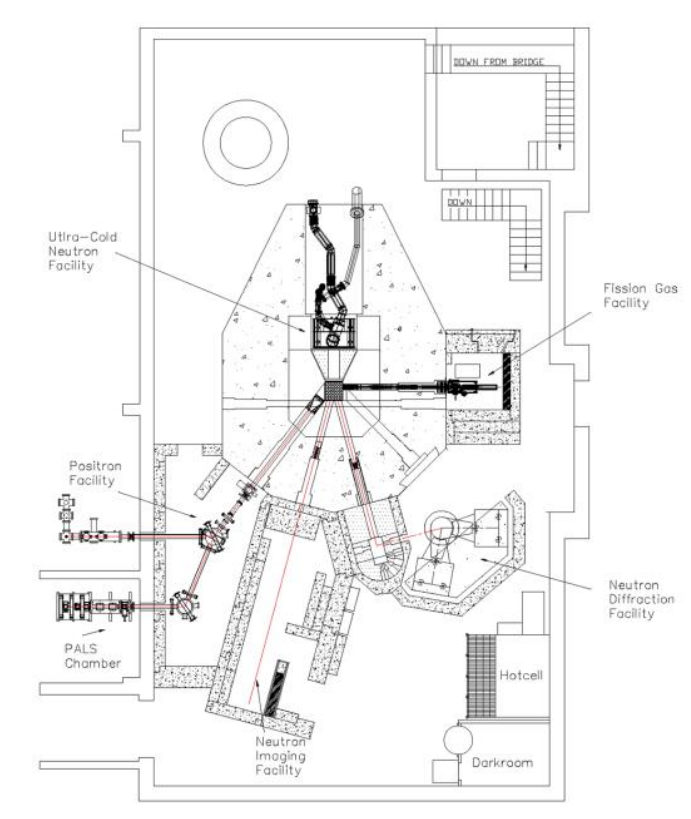

Fig. 1. PULSTAR bay floor and beamport experimental facilities.

\subsection{History of Education and Training}

Since its initial criticality in 1972, the PULSTAR Reactor has been utilized to provide instructional and training content to nuclear engineers and reactor operators from academia and industry. Undergraduate students in the Department of Nuclear Engineering utilize the facility for reactor kinetics and thermal hydraulic academic laboratories, and frequently as the focus for senior design projects. The NE235 Nuclear Reactor Operations training course provides hand-on operations experience for undergraduates interested in becoming reactor operators, with several students earning NRC reactor operator licenses each year. Plant operators from local nuclear utilities utilized the facility through the late 1990's to perform required operations training evolutions in support of their NRC approved requalification programs.

Under the DOE Innovations in Nuclear Infrastructure and Education (INIE) Program in 2002, N.C. State University was awarded a project to install distance learning capabilities at the PULSTAR Reactor with the intent of enriching educational experiences for nuclear engineering students at academic institutions without a research reactor of their own. The vision was to enable remote students to experience and participate in reactor laboratory sessions as if they were in the control room observing and taking data firsthand. An RTP Micro 2000 data acquisition system was selected to provide remote distribution of live reactor telemetry given widespread experience in the nuclear industry with this hardware and vendor. 110 channels of reactor instrumentation were connected, including power monitoring channels with level and period indications, control rod positions, primary coolant temperature, level, and flow, SCRAM logic outputs and annunciator indications, and various experimental instrumentation including in-core flux monitor detectors and thermocouples. Given operational safety and cyber-security concerns, reactor system control functions were not connected to this system. An associated NetSuite software package allowed the configuration of a GUI that closely resembled the instrumentation readouts on the PULSTAR reactor console (see Fig. 2). Remote users installed an executable file on their PC that established a real time network connection to the Micro 2000 unit server, and provided display of live reactor telemetry via the console GUI. A Polycom teleconferencing unit was installed in the PULSTAR control room with a PTZ camera and room microphone. This system allowed remote users to interact via a live feed with the facility operations staff, and remotely direct the camera to view console instrumentation and other content of interest. 


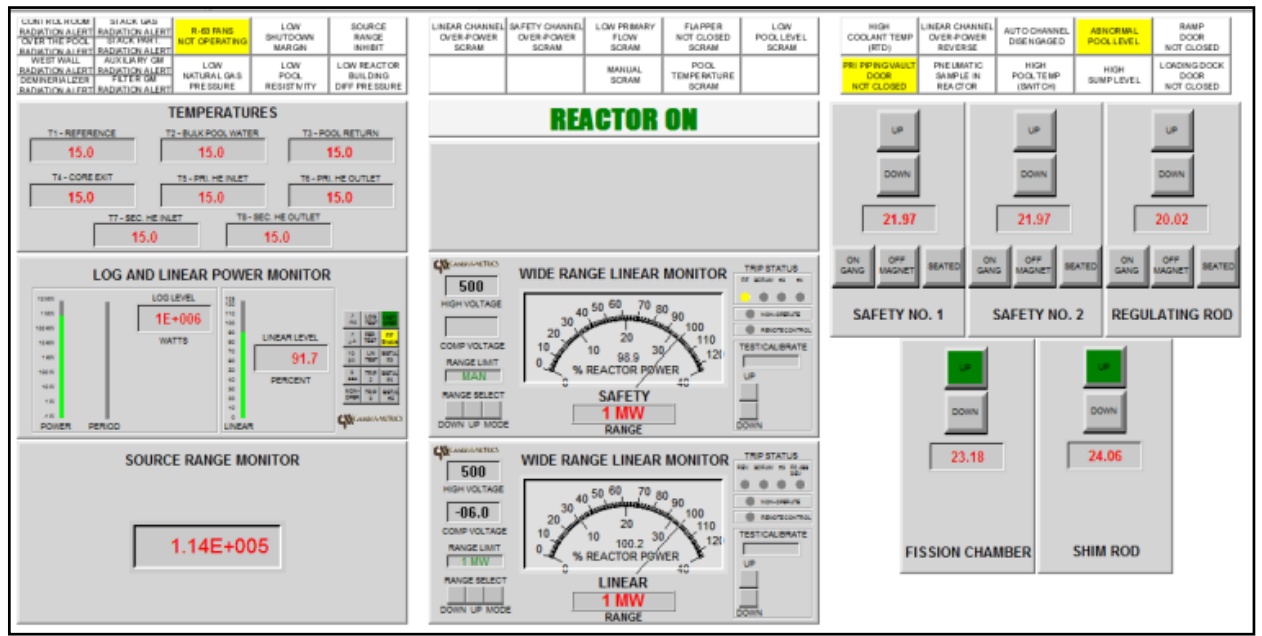

Fig. 2. Original first generation NetSuite PULSTAR console GUI.

The original system infrastructure was utilized to provide internet reactor laboratory sessions to external academic users including the University of Tennessee at Knoxville Dept. of Nuclear Engineering, the Georgia Institute of Technology Dept. of Nuclear \& Radiological Engineering, and the Jordan University of Science and Technology Dept. of Nuclear Engineering (see Fig. 3) [4]. Forty-five separate IRL sessions were provided during the period from 2004 through 2013 to hundreds of nuclear engineering students at these universities.

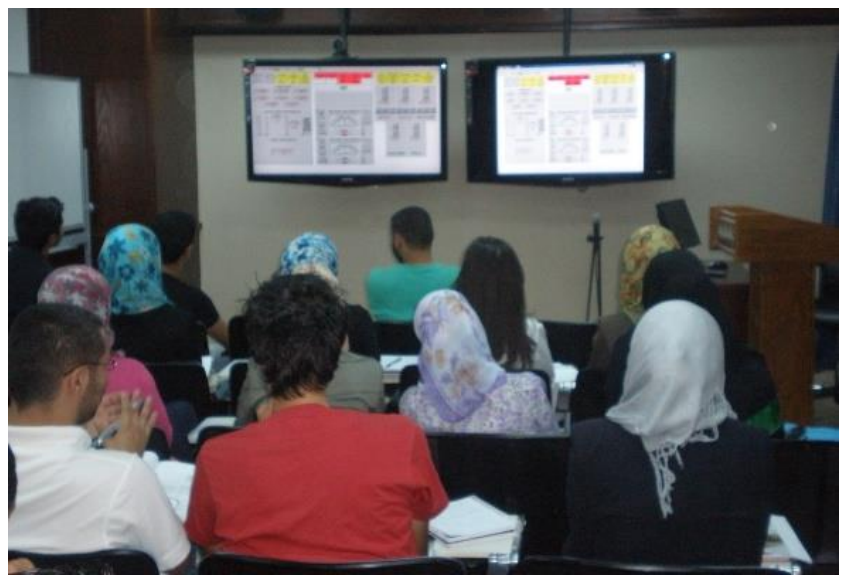

Fig. 3. Remote reactor laboratory session held on the campus of the Jordan University of Science and Technology.

\section{STATE-OF-THE-ART INTERNET REACTOR LABORATORY INFRASTRUCTURE UPGRADES}

Under Section 123 of the Atomic Energy Act, Vietnam and the United States entered into an Administrative Arrangement in 2016. One of the key objectives of the arrangement was to enhance nuclear engineering education and reactor operations training opportunities for Vietnamese students. Consequently, the NRP was awarded funding to upgrade its IRL infrastructure to incorporate new state-of-the-art platforms for relaying live reactor telemetry data and video-teleconferencing connections internationally. The goal was to provide a realistic laboratory experience to remote students, allowing them to interact with the PULSTAR reactor operators and view, record, and process live reactor data in real time. In designing the upgraded 
systems, therefore, the following functional objectives were set: 1) provide robust, easy-to-use, and cybersecure graphical user interfaces allowing students to stream, observe and record reactor channel output data in real time; 2) provide customized GUIs for each laboratory module focusing on relevant channels and linking to supporting documentation and schematics; and 3) provide video teleconferencing capabilities supporting content sharing across multiple remote user platforms including tablets and smartphones. Installation of the upgraded IRL hardware and software platforms were completed and commissioned in 2018.

\subsection{IRL System Hardware}

Cisco was selected as the vendor for the video-teleconferencing system, with a SX80 Codec providing the nexus for the installed Telepresence system. The Telepresence system is capable of connecting to remote sites using multiple platforms, including room systems, PC/laptops, tablets and smartphones. This functionally diverse platform is useful in providing a variety of options when connecting to remote sites which may have limited teleconferencing infrastructure. Two high definition pan-tilt-zoom (PTZ) cameras mounted in the control room along with audio equipment provide for live interaction between the remote site and the PULSTAR reactor staff (see Fig. 4). The cameras, local 65" and 75" touch screen displays and a graphics PC are connected to a Touch Tablet, which allows a presenter to control and display video and graphical laboratory content locally and remotely.

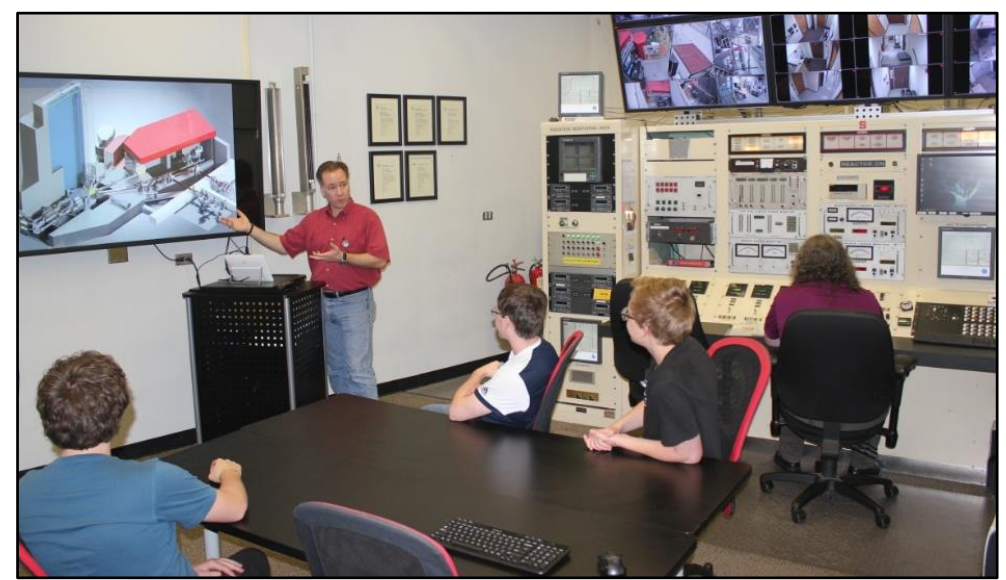

Fig. 4. Cisco telepresence camera view in PULSTAR reactor control room

National Instruments (NI) was selected as the vendor for the data acquisition system hardware, with LabView software providing the digital user interface. The NI data acquisition modules accept optically isolated digital, analog, and thermocouple inputs from the reactor control, monitoring, and experimental systems. Given cyber-security and operations safety concerns, no data output cards are installed in the system and no reactor control functions are connected. 78 digital inputs monitor the discrete ON/OFF states of reactor instrumentation such as coolant pumps, control rod limit switches, channel annunciators and alarms, and SCRAM signals. 42 analog inputs monitor continuous signal outputs of channels such as reactor power level, control rod height, coolant flow rate, coolant system temperatures, and in-core miniature fission chamber output. Ten thermocouple inputs are connected to K-type temperature sensors which may be inserted into the fuel coolant channels to measure distributed in-core temperature profiles.

\subsection{IRL System Software Interface}

The NI hardware interface input cards described above are connected to a customized LabView data acquisition system running on a local Windows PC based server. The local data acquisition server is 
capable of streaming live data to one hundred remote student users simultaneously. The remote student user downloads a LabView data acquisition executable file from cloud-based storage and installs it on their computer. For each IRL session, remote students are provided with unique credentials allowing secure encrypted connection to the server. Once logged in, the student's application connects to the local data acquisition server and streams the live reactor telemetry to their desktop. The LabView application GUI have been designed to display 130 channels of live reactor telemetry represented by realistic depictions of instrumentation installed in the reactor console (see Fig. 5). Students can view, record and process the data live on their remote computer, just as they would if they were participating in a laboratory session on site.

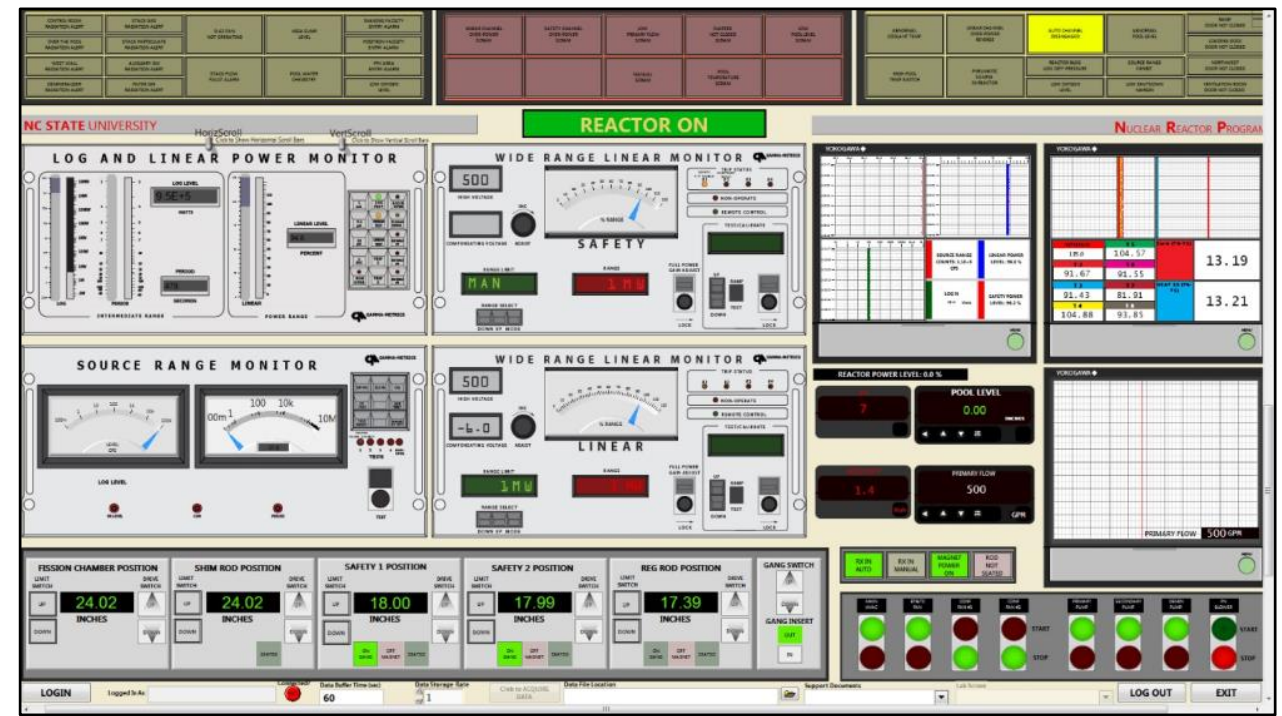

Fig. 5. PULSTAR Main Console LabView GUI displaying live reactor telemetry including power levels, control rod positions, coolant temperature and flow, and equipment status indiations.

Additional GUIs have been customized with graphics to support specific laboratory sessions, and have supporting content integrated such as schematics and drawings that illustrate associated technical details and concepts. Examples of integrate supporting content include a 3D facility model, a fuel assembly diagram (see Fig. 6), a control rod diagram, a cooling system schematic with live temperature telemetry, and a reactor facility elevation view. Following the completion of each IRL session, the remote student has the ability to process and analyze the reactor telemetry data they have recorded.

\section{INTERNET REACTOR LABORATORY SESSIONS}

A selection of nine different remote internet reactor laboratory sessions are offered which are identical in nature to those provided to nuclear engineering students on site at N.C. State University. IRL sessions offered include

1. Introduction to Reactor Systems: Utilizing the main console GUI (see Fig. 5) and supporting content (such as Figure 6), students are introduced to the reactor plant systems. Reactor core components and configuration, reactor safety channels with console instrumentation, SCRAM logic and safety interlocks, reactor coolant systems, radiological safety systems and experimental systems are all discussed. Presentation of system layouts is aided by reactor staff walking down the reactor facility systems while streaming live video and audio from a handheld device to the Telepresence system. 


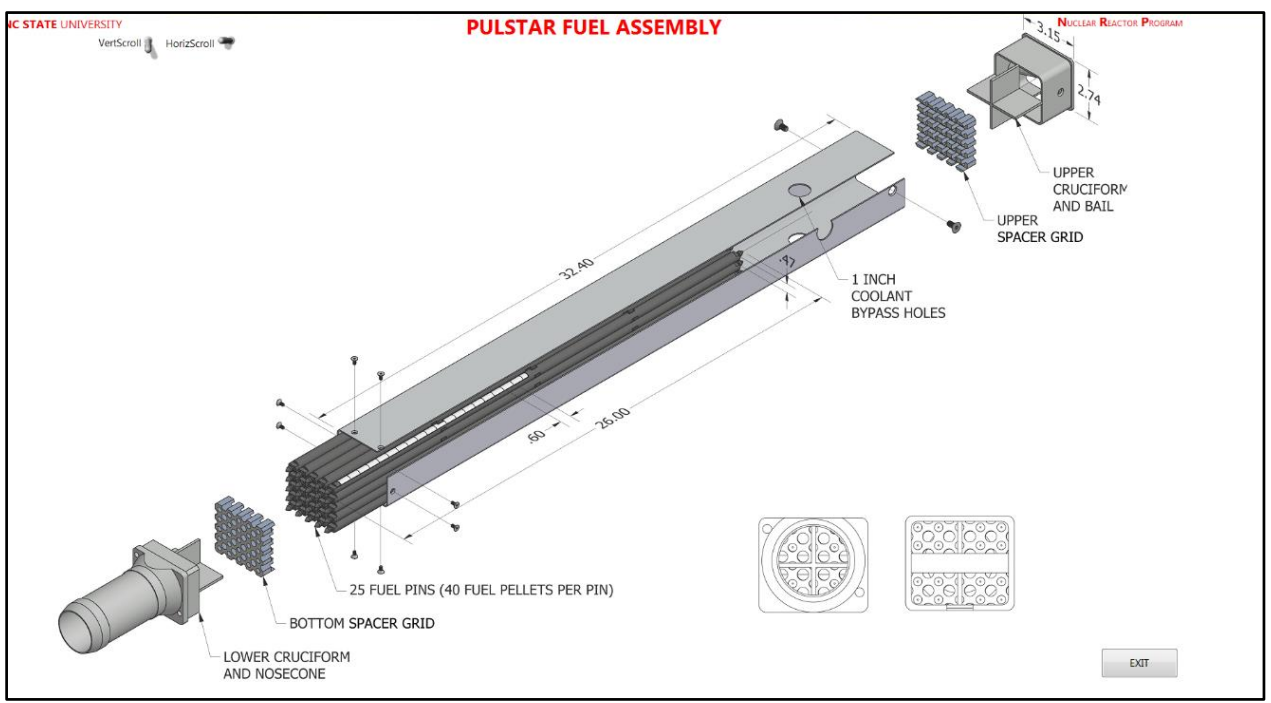

Fig. 6. Diagram of PULSTAR fuel assembly.

2. 1/M Approach to Criticality: Utilizing a GUI detailing the startup range instrumentation and control rod positions, students take the reactor from shutdown to criticality utilizing a conservative 1/M startup methodology. Utilizing the count rate from the Source Range Monitor, students make conservative projections of the critical control rod height, iterating until criticality is achieved.

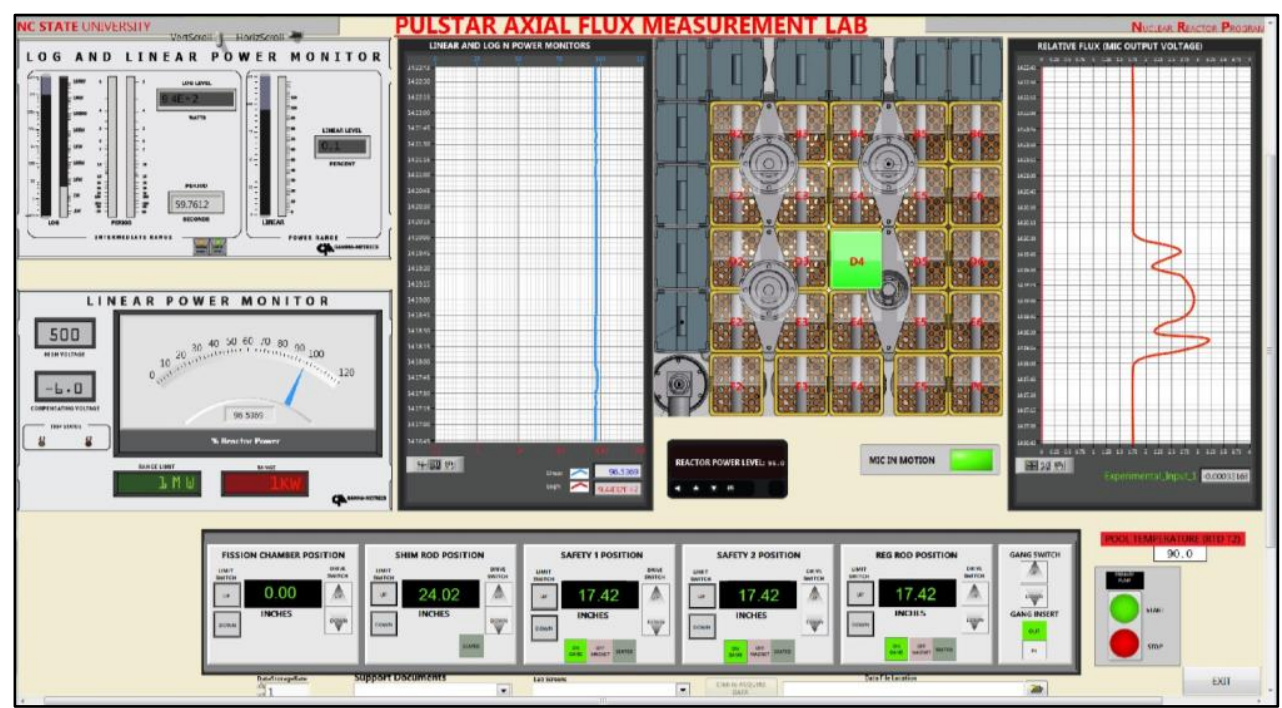

Fig. 7. Axial Flux Measurement Laboratory GUI; note the position of the dry well highlighted in green in fuel assembly D4, and the detector current trace at right.

3. Normal Reactor Startup and Power Range Operations: Starting with the reactor in a shutdown state, students compute an estimated critical rod position by performing a reactivity balance. The reactor is then brought to criticality at low power through following operations procedures and observing the characteristics of subcritical multiplication on the power monitoring channels presented on the main console GUI. The reactor is then raised to higher power levels, with students observing the response of instrumentation and temperature feedback characteristics.

4. Control Rod Calibration: A reactor control rod is calibrated utilizing the constant period method. The reactor is brought critical at low power with the rod to be calibrated inserted. The rod is then withdrawn 
in steps, and students measure the power doubling times through observing the response of the Linear Power Channel via a customized laboratory GUI. Students then calculate the reactor period for each step and plot the differential and integral rod worth curves as a function of control rod height.

5. Axial Flux Measurement: A miniature in-core (MIC) fission chamber neutron detector is inserted in a dry well placed inside a PULSTAR fuel assembly coolant channel. The reactor is operated at low power while the detector is slowly withdrawn along the axis of the cooling channel, measuring the neutron flux profile as a function of core height (see trace in Figure 7 at right). This measurement is repeated for five fuel assemblies in a row across the core to allow students to calculate the radial and axial core neutron flux distributions.

6. Power Coefficient Measurement: The reactor power coefficient is measured dynamically through inserting a known amount of positive reactivity into the reactor from criticality at low power. The resulting power transient is observed and recorded utilizing the main console GUI (see Figure 5, power and coolant temperature recorders indicating at right). The time dependent power and moderator temperature data may then be then utilized by students to calculate the power coefficient of reactivity.

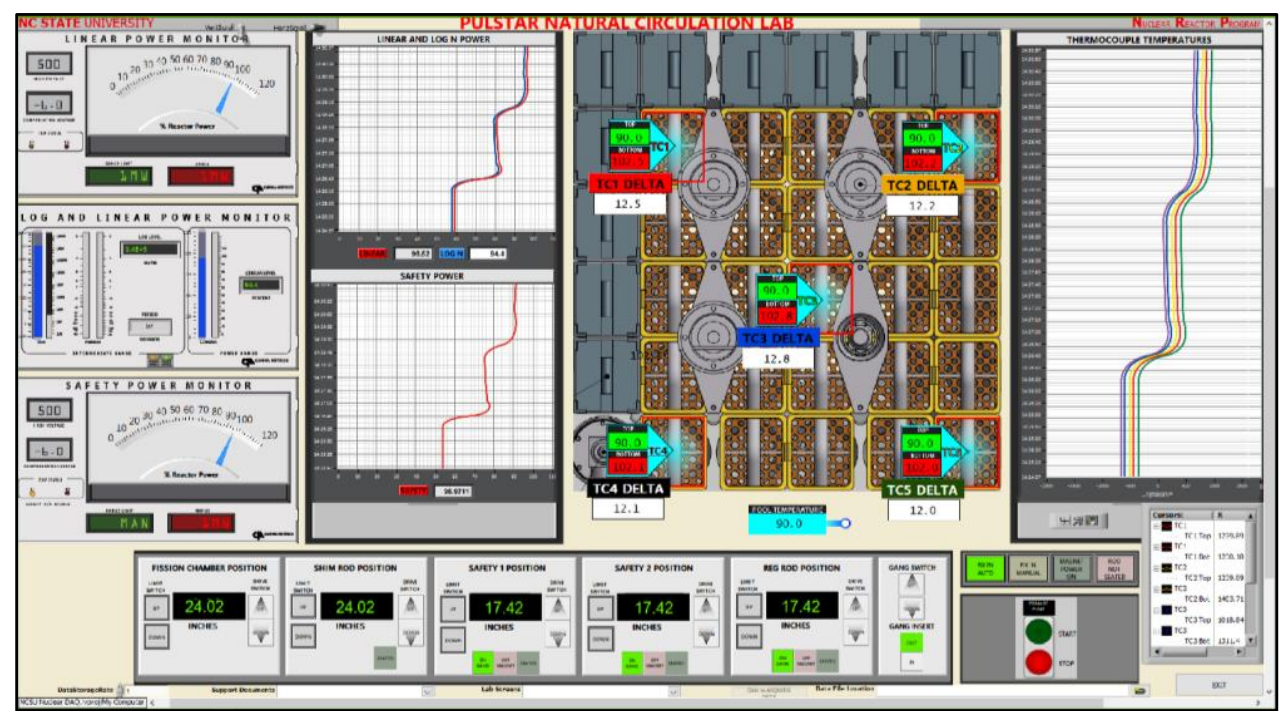

Fig. 8. Natural Circulation Laboratory GUI; note in-core thermocouple positions with instantaneous temperature readouts, power level traces at center left, and transient coolant channel temperature traces at right.

7. Void Coefficient Measurement: The reactor is brought critical at low power and an empty dry well of known volume is placed inside a PULSTAR fuel assembly coolant channel to displace the moderator. The resulting change in reactivity is measured through observing reactor control rod height on the main console GUI and adjusting the control rods to maintain criticality. This measurement is repeated for five fuel assemblies in a row across the core to allow students to determine spatial dependency.

8. Heat Balance Power Calibration: A heat balance is performed by bringing the reactor critical at several power levels ranging from $10 \%$ to $100 \%$ of full power. Students observe the primary coolant temperature and flow channel telemetry presented on the main console GUI (see Figure 5, temperature recorder at right). Temperature changes across the core and primary heat exchanger are measured at each power level and utilized to perform a heat balance calculation.

9. Natural Circulation / Flow Reversal: Dry wells containing two thermocouples each (positioned at the top and bottom of the fuel) are inserted into five fuel assembly coolant channels located at the corners 
and center of the PULSTAR core. The reactor is operated at low power under forced downward primary flow cooling conditions and allowed to come to thermal equilibrium. The primary coolant pump is then tripped and core cooling flow coasts to a stop and then reverses as natural convection flow is established. Utilizing the natural circulation laboratory GUI (see Fig. 8), students observe and measure the coolant channel temperatures during steady state conditions and the flow reversal transient.

\section{CONCLUSIONS}

"Second generation" IRL data acquisition and video-teleconferencing systems have been commissioned at the NCSU PULSTAR Reactor Facility. The NRP has offered IRL based reactor physics experiments and training since 2004, enriching the academic programs at its user universities, and meeting the objective of expanding educational opportunities for nuclear engineering students in the United States and internationally. The N.C. State IRL model has been adopted by the International Atomic Energy Agency (IAEA), which has funded member states in South America, Europe, Asia and Africa to develop additional IRL capabilities to serve engineering students at academic institutions around the world [5]. With its upgraded state-of-the-art data acquisition and teleconferencing system infrastructure, NCSU is able to provide improved IRL experiences to engineering students in at universities located in the U.S. and internationally.

\section{ACKNOWLEDGEMENTS}

The Nuclear Reactor Program acknowledges the U.S. Department of Energy for funding the upgrade of IRL infrastructure at the North Carolina State University PULSTAR Reactor facility.

\section{REFERENCES}

1. A. I. Hawari, "Multidisciplinary Engagement at Research Reactors: The NCSU PULSTAR," IGORR 2017, Sydney, Australia (2017).

2. A. I. Hawari et al, "Implementation of a Fission Gas Release and Monitoring Loop at the PULSTAR Reactor," IGORR 2016, Berlin, Germany, 2016.

3. A. I. Hawari, "PULSTAR Reactor Power Upgrade from 1-MWth to 2-MWth," IGORR 2013 Conference, Daejeon, South Korea, 2013.

4. S. Malkawi, A. I. Hawari, A. Obeidat and O. Al-Araidah, "Exploring the utilization of nuclear research reactors in distance education across international borders," 2010 1st International Nuclear \& Renewable Energy Conference (INREC), Amman, 2010, pp. 1-5. doi: 10.1109/INREC.2010.5462589

5. "The IAEA Internet Reactor Laboratory Programme (IRL) is Expanding to Africa", https://www.iaea.org/newscenter/news/the-iaea-internet-reactor-laboratory-irl-programme-isexpanding-to-africa (2017). 